\title{
BLOW-UP OF $H^{1}$ SOLUTIONS FOR THE ONE-DIMENSIONAL NONLINEAR SCHRÖDINGER EQUATION WITH CRITICAL POWER NONLINEARITY
}

\author{
TAKAYOSHI OGAWA AND YOSHIO TSUTSUMI \\ (Communicated by George C. Papanicolaou)
}

\begin{abstract}
We investigate the blow-up of solutions in $H^{1}(\mathbf{R})$ with negative energy for the one-dimensional nonlinear Schrödinger equation with critical power nonlinearity:

$$
\begin{gathered}
i u_{t}=-u_{x x}-|u|^{4} u, \quad t>0, x \in \mathbf{R}, \\
u(0, x)=u_{0}(x), \quad x \in \mathbf{R} .
\end{gathered}
$$

In our result we remove the weight condition $x u_{0} \in L^{2}(\mathbf{R})$, which was always assumed to show the blow-up of solutions in the previous papers.
\end{abstract}

\section{INTRODUCTION AND A THEOREM}

In the present paper we consider the blow-up problem of solutions for the onedimensional nonlinear Schrödinger equation with critical power nonlinearity:

$$
\begin{gathered}
i \frac{\partial u}{\partial t}=-D^{2} u-|u|^{4} u, \quad t>0, x \in \mathbf{R}, \\
u(0, x)=u_{0}(x),
\end{gathered}
$$

where $D$ denotes $\partial / \partial x$.

When the nonlinear term is $-|u|^{p-1} u$, it is well known that if $p<5$ all the solutions in $H^{1}(\mathbb{R})$ exist globally in time, and that if $p \geq 5$ there exist blowup solutions for certain initial data (see Glassey [5] and M. Tsutsumi [19]). Accordingly, the power of 5 is called the critical power. Equation (1.1) is of physical interest because it describes the collapse of a plane plasma soliton (see [3]). Recently, many mathematicians have been studying the behavior of blowup solutions near blow-up time for (1.1)-(1.2) (see [2], [8], [9], [1 i ]-[15], [18], and [20]-[23]). Most of these results are considered in the framework of $H^{1}(\mathbb{R})$.

Received by the editors November 30, 1989.

1980 Mathematics Subject Classification (1985 Revision). Primary 35B40; Secondary 35Q20, $35 \mathrm{~L} 15$.

The second author is partially supported by a Grant-in-Aid for Encouragement of Young Scientists. 
Nevertheless, the blow-up of solutions for (1.1)-(1.2) has been proved only in the framework of $H^{1}(\mathbb{R}) \cap L^{2}\left(\mathbb{R} ;|x|^{2} d x\right)$. The proofs in the previous papers are based on the pseudoconformal conservation law, which is satisfied by the solution in $H^{1}(\mathbb{R}) \cap L^{2}\left(\mathbb{R} ;|x|^{2} d x\right)$ (see [4], [5], and [19]). When we consider the existence or nonexistence of global solutions for $(1.1)-(1.2), H^{1}(\mathbb{R})$ seems to be more natural than $H^{1}(\mathbb{R}) \cap L^{2}\left(\mathbb{R} ;|x|^{2} d x\right)$. In [16] the authors showed that if the spatial dimensions $N \geq 2$ and the nonlinear term is $-|u|^{p-1} u$ for $1+4 / N \leq p<\min \{5,(N+2) /(N-2)\}$, then all the radially symmetric solutions in $H^{1}\left(\mathbb{R}^{N}\right)$ of $(1.1)-(1.2)$ with negative energy must blow up in finite time. In the above direction, this is an improvement of the results due to Glassey [5] and $M$. Tsutsumi [19] for the radially symmetric solution.

However, the proof in [16] is based on the decay property of the radially symmetric function; that is, if $v(x)=v(|x|), v \in H^{1}\left(\mathbb{R}^{N}\right)$ and $N \geq 2, v(x)$ decays like $|x|^{-(N-1) / 2}$ as $|x| \rightarrow \infty$. Therefore, the proof in [16] is not directly applicable to the one-dimensional case. But, fortunately (1.1) has one more symmetry than the supercritical case (see, e.g., [15] and [22]). Combining the proof in [16] and the scaling argument, we can drop the weight restriction of $L^{2}\left(\mathbb{R} ;|x|^{2} d x\right)$ for $(1.1)-(1.2)$. We then have the following theorem:

Theorem. Assume that $u_{0} \in H^{1}(\mathbb{R})$ and that

$$
E\left(u_{0}\right)=\left\|D u_{0}\right\|_{L^{2}}^{2}-\frac{1}{3}\left\|u_{0}\right\|_{L^{6}}^{6}<0 .
$$

Then, the solution $u(t)$ in $H^{1}(\mathbb{R})$ of $(1.1)-(1.2)$ blows up in finite time; that is, for some finite $T>0$,

$$
\|D u(t)\|_{L^{2}} \rightarrow \infty \quad(t \rightarrow T) .
$$

Remark. (1) The unique local existence theorem in $H^{1}(\mathbb{R})$ for $(1.1)-(1.2)$ is already established: If $u_{0} \in H^{1}(\mathbb{R})$, then there exists a unique solution $u(t)$ of (1.1)-(1.2) in $C\left([0, T) ; H^{1}(\mathbb{R})\right)$ for some $T>0$, and $u(t)$ satisfies two conservation laws of the $L^{2}$ norm and the energy

$$
\begin{aligned}
\|u(t)\|_{L^{2}} & =\left\|u_{0}\right\|_{L^{2}}, \\
E(u(t)) & =E\left(u_{0}\right),
\end{aligned}
$$

for $0 \leq t<T$. Moreover, $T=\infty$ or $T<\infty$ and $\|D u(t)\|_{L^{2}} \rightarrow \infty(t \rightarrow T)$. For the details, see, e.g., Ginibre and Velo [4], Kato [6], and Cazenave and Weissler [1].

(2) If $E\left(u_{0}\right) \geq 0$, then the solution in $H^{1}(\mathbb{R})$ of $(1.1)-(1.2)$ does not always blow up in finite time. It is known that there exists a standing-wave solution with $E\left(u_{0}\right)=0$ (see, e.g., Weinstein [21], [22] and Strauss [17], [18]).

(3) In the above theorem, the weight condition $x u_{0} \in L^{2}(\mathbb{R})$ is not needed, which is suggested through the numerical computation (see $[9, \S 5])$.

(4) The proofs of blow-up in [5] and [19] give the upper bound of the blow-up time dcpending on $\left\|u_{0}\right\|_{H^{1}}, E\left(u_{0}\right)$, and $\left\|x u_{0}\right\|_{L^{2}}$. That upper bound goes to 
infinity as $\left\|x u_{0}\right\|_{L^{2}} \rightarrow \infty$. However, our proof of the theorem gives the upper bound of the blow-up time depending on $\left\|u_{0}\right\|_{H^{1}}, E\left(u_{0}\right)$ and the distribution of the initial $L^{2}$ density, which is finite even for the initial data $u_{0}$ with $\left\|x u_{0}\right\|_{L^{2}}=$ $\infty$.

Our plan in this paper is as follows. In $\S 2$ we prepare three lemmas needed for the proof of the theorem. In $\S 3$ we give the proof of the theorem.

We conclude this section by giving several notations. Throughout this paper we omit the integral region, when it is the whole real line $\mathbb{R}$. We abbreviate $L^{q}(\mathbb{R})$ and $H^{m}(\mathbb{R})$ to $L^{q}$ and $H^{m}$, respectively. We denote the $L^{q}$ norm by $\|\cdot\|_{q}$. Let $W^{m, \infty}=\left\{f \in L^{\infty} ; D^{j} f \in L^{\infty}, 1 \leq j \leq m\right\}$ for a positive integer $m$. For $z \in \mathbb{C}$ we denote the complex conjugate of $z$ by $\bar{z}$.

\section{LEMMAS}

In this section we give three lemmas needed for the proof of the theorem. The first is a variant of the Gagliardo-Nirenberg inequality.

Lemma 2.1. Let $u \in H^{1}$, and let $\rho$ be a real-valued function in $W^{1, \infty}$. Then, for any $r>0$, we have

$$
\begin{aligned}
\|\rho u\|_{L^{\infty}(|x|>r)} \leq & \|u\|_{L^{2}(|x|>r)}^{1 / 2}\left[2\left\|\rho^{2} D u\right\|_{L^{2}(|x|>r)}\right. \\
& \left.+\left\|u D \rho^{2}\right\|_{L^{2}(|x|>r)}\right]^{1 / 2} .
\end{aligned}
$$

Proof. It suffices to show Lemma 2.1 for $u \in C_{0}^{\infty}(\mathbb{R})$. Lemma 2.1 for the general case can be proved by using the standard regularizing argument. For $x>r$, we have

$$
\begin{aligned}
|u(x)|^{2} \rho(x)^{2} & =-\int_{x}^{\infty} D\left(|u(y)|^{2} \rho(y)^{2}\right) d y \\
& \leq \int_{r}^{\infty}|u|^{2}\left|D \rho^{2}\right| d y+2 \int_{r}^{\infty}\left|\rho^{2} u D u\right| d y .
\end{aligned}
$$

For $x<-r$, we also have

$$
|u(x)|^{2} \rho(x)^{2} \leq 2 \int_{-\infty}^{-r}\left|\rho^{2} u D u\right| d y+\int_{-\infty}^{-r}|u|^{2}\left|D \rho^{2}\right| d y .
$$

Combining (2.2) and (2.3), we obtain

$$
\begin{aligned}
|u(x)|^{2} \rho(x)^{2} & \leq 2 \int_{|x|>r}\left|\rho^{2} u D u\right| d y+\int_{|x|>r}\left|u^{2} D \rho^{2}\right| d y \\
& \leq\|u\|_{L^{2}(|x|>r)}\left[2\left\|\rho^{2} D u\right\|_{L^{2}(|x|>r)}+\left\|u D \rho^{2}\right\|_{L^{2}(|x|>r)}\right],
\end{aligned}
$$

which completes the proof. Q.E.D.

The following lemma is a generalization of the identities obtained by Glassey [5] (see also Lin and Strauss [10] and Kavian [8]). 
Lemma 2.2. Let $0<T \leq \infty, u(t)$ be a solution of $(1.1)-(1.2)$ in $C\left([0, T) ; H^{1}\right)$, and $\varphi$ be a real-valued function in $W^{3, \infty}$ with compact support. We put $\Phi(x)=\int_{0}^{x} \varphi(y) d y$. Then we have

$$
\begin{aligned}
&-\operatorname{Im} \int \varphi u(t) D \bar{u}(t) d x+\operatorname{Im} \int \varphi u_{0} D \bar{u}_{0} d x \\
&=\int_{0}^{t}\left[2 \int D \varphi|D u(s)|^{2} d x\right.-\frac{2}{3} \int D \varphi|u(s)|^{6} d x \\
&\left.-\frac{1}{2} \int D^{3} \varphi|u(s)|^{2} d x\right] d s,
\end{aligned}
$$

$$
\int \Phi|u(t)|^{2} d x=\int \Phi\left|u_{0}\right|^{2} d x-2 \int_{0}^{t} \operatorname{Im} \int \varphi u(s) D \bar{u}(s) d x d s
$$

for $0 \leq t<T$.

Proof. We briefly state the formal calculations of the proof. For the details, see [16, Lemma 2.2 and (4.9)] (see also Kavian [8, Lemma 2.9]).

Let $u(t, x)$ be a smooth solution of (1.1)-(1.2). We first show (2.4). We multiply (1.1) by $\varphi D \bar{u}$ and take the real part to obtain

$$
\begin{aligned}
-\frac{d}{d t} & \operatorname{Im} \int \varphi u(t) D \bar{u}(t) d x-\operatorname{Im} \int D \varphi u(t) \bar{u}_{t}(t) d x \\
& =\int D \varphi|D u(t)|^{2} d x+\frac{1}{3} \int D \varphi|u(t)|^{6} d x .
\end{aligned}
$$

We next multiply the complex conjugate of $(1.1)$ by $D \varphi u$ and take the real part to obtain

$$
\begin{aligned}
\operatorname{Im} \int D \varphi u(t) \bar{u}_{t}(t) d x= & \int D \varphi|D u(t)|^{2} d x-\int D \varphi|u(t)|^{6} d x \\
& -\frac{1}{2} \int D^{3} \varphi|u(t)|^{2} d x .
\end{aligned}
$$

(2.6) and (2.7) give us (2.4).

We next multiply the complex conjugate of (1.1) by $\Phi u$ and take the imaginary part to obtain (2.5). Q.E.D.

We conclude this section by giving the following lemma:

Lemma 2.3. Let $v(x)$ be in $L^{2}$. We define $R(x)$ by

$$
R(x)= \begin{cases}|x|, & |x|<1 \\ 1, & |x|>1\end{cases}
$$

We put $v_{\varepsilon}(x)=\varepsilon^{-1 / 2} v(x / \varepsilon)$, for $\varepsilon>0$. Then, for any $\delta>0$, there exists an $\varepsilon_{0}>0$ such that $\left\|R v_{\varepsilon}\right\|_{2} \leq \delta$ for $0<\varepsilon<\varepsilon_{0}$.

Proof. For any $\delta>0$, there exists an $r>0$ such that $\|v\|_{L^{2}(|x|>r)} \leq \frac{1}{2} \delta$. 
Therefore, if we choose $\varepsilon_{0}=\min \left\{\delta\left(2 r\|v\|_{2}\right)^{-1}, 1 / r\right\}$, then we have

$$
\begin{aligned}
\left\|R v_{\varepsilon}\right\|_{L^{2}(|x|>\varepsilon r)} & \leq\left\|v_{\varepsilon}\right\|_{L^{2}(|x|>\varepsilon r)}=\|v\|_{L^{2}(|x|>r)} \leq \frac{1}{2} \delta, \\
\left\|R v_{\varepsilon}\right\|_{L^{2}(|x|<\varepsilon r)} & =\left\|x v_{\varepsilon}\right\|_{L^{2}(|x|<\varepsilon r)} \leq \varepsilon r\left\|v_{\varepsilon}\right\|_{L^{2}(|x|<\varepsilon r)} \\
& \leq \varepsilon r\|v\|_{2} \leq \frac{1}{2} \delta,
\end{aligned}
$$

for $0<\varepsilon<\varepsilon_{0}$. These complete the proof of Lemma 2.3. Q.E.D.

\section{PROOF OF THEOREM}

In this section we prove the theorem. For that purpose, we first show that if $-E\left(u_{0}\right)$ is large and $\left\|u_{0}\right\|_{L^{2}(|x|>1)}$ is small (but $\left\|u_{0}\right\|_{L^{2}(|x|<1)}$ may be large), then $\|u(t)\|_{L^{2}(|x|>1)}$ is small for all $t>0$, that is, $u(t)$ is localized around the origin, as long as $u(t)$ exists. Roughly speaking, this fact implies that under the above conditions the nonlinear effect is stronger than the dispersive effect. This leads to the blow-up of solutions. We next extend this result to the general initial data with negative energy by the scaling argument. Since (1.1) has the critical power nonlinearity, the solution $u(t)$ of (1.1) has the following scaling invariance. If we put

$$
u_{\varepsilon}(t, x)=\varepsilon^{-1 / 2} u\left(t / \varepsilon^{2}, x / \varepsilon\right)
$$

for $\varepsilon>0$, then $u_{\varepsilon}(t, x)$ also satisfies (1.1) with initial cata $u_{0 \varepsilon}(x)=\varepsilon^{-1 / 2}$ $\times u_{0}(x / \varepsilon)$. For any initial data $u_{0}$ with negative energy, we can choose $\varepsilon>0$ so small that $-E\left(u_{0 \varepsilon}\right)$ is sufficiently large and $\left\|u_{0 \varepsilon}\right\|_{L^{2}(|x|>1)}$ is sufficiently small. But we note that $\left\|u_{0 \varepsilon}\right\|_{2}=\left\|u_{0}\right\|_{2}$. This observation enables us to prove the blow-up of solutions for any initial data with negative energy.

Let $\varphi(r)$ be a real-valued function in $W^{3, \infty}$ such that

$$
\varphi(x)= \begin{cases}x, & 0<|x|<1, \\ x-(x-1)^{3}, & 1+1 / \sqrt{3}>x>1, \\ x-(x+1)^{3}, & -(1+1 / \sqrt{3})<x<-1, \\ \text { smooth, } & 1+1 / \sqrt{3}<|x|<2, \\ 0, & |x|>2\end{cases}
$$

and $\varphi^{\prime}(x) \leq 0$ for $|x|>1+1 / \sqrt{3}$. We put $\Phi(x)=\int_{0}^{x} \varphi(y) d y$. Let $E_{0}=$ $E\left(u_{0}\right)$.

Before we state the proof of the theorem, we show the following proposition.

Proposition 3.1. Let $0<T \leq \infty$ and let $u(t)$ be a solution of (1.1)-(1.2) in $C\left([0, T) ; H^{1}\right)$. Put $a_{0}=(3 / 8)^{1 / 4}$. If $u(t)$ satisfies

$$
\|u(t)\|_{L^{2}(|x|>1)} \leq a_{0}, \quad 0 \leq t<T,
$$

then we have

$$
\begin{aligned}
& -\operatorname{Im} \int \varphi u(t) D \bar{u}(t) d x+\operatorname{Im} \int \varphi u_{0} D \bar{u}_{0} d x \\
& \quad \leq\left[2 E_{0}+4(1+M)^{2}\left\|u_{0}\right\|_{2}^{6}+\frac{M}{2}\left\|u_{0}\right\|_{2}^{2}\right] t, \quad 0 \leq t<T,
\end{aligned}
$$

where $M=\left\|D^{2} \varphi\right\|_{\infty}+\left\|D^{3} \varphi\right\|_{\infty}$. 
Proof. Since we have, by (1.4),

$$
\int_{|x|<1}|D u(t)|^{2} d x=E_{0}-\int_{|x|>1}|D u(t)|^{2} d x+\frac{1}{3} \int|u(t)|^{6} d x
$$

we obtain by (2.4) and (3.2)

$$
\begin{aligned}
-\operatorname{Im} \int \varphi u(t) D \bar{u}(t) d x+\operatorname{Im} \int \varphi u_{0} D \bar{u}_{0} d x \\
=\int_{0}^{t}\left[2 \int D \varphi|D u(s)|^{2} d x-\frac{2}{3} \int D \varphi|u(s)|^{6} d x-\frac{1}{2} \int D^{3} \varphi|u(s)|^{2} d x\right] d s \\
=\int_{0}^{t}\left[2 E_{0}-2 \int_{|x|>1}(1-D \varphi)|D u(s)|^{2} d x\right. \\
\left.\quad+\frac{2}{3} \int_{|x|>1}(1-D \varphi)|u(s)|^{6} d x-\frac{1}{2} \int D^{3} \varphi|u(s)|^{2} d x\right] d s .
\end{aligned}
$$

We choose $\rho(x)=(1-D \varphi(x))^{1 / 4}$ in Lemma 2.1, and use Lemma 2.1 and Hölder's inequality to obtain

$$
\begin{aligned}
& \int_{|x|>1}(1-D \varphi)|u|^{6} d x \leq\|u\|_{L^{2}(|x|>1)}^{2}\|\rho u\|_{L^{\infty}(|x|>1)}^{4} \\
& \quad \leq\|u\|_{L^{2}(|x|>1)}^{4}\left[2\left\|\rho^{2} D u\right\|_{L^{2}(|x|>1)}+\left\|u D \rho^{2}\right\|_{L^{2}(|x|>1)}\right]^{2} \\
& \quad \leq 8\|u\|_{L^{2}(|x|>1)}^{4}\left\|\rho^{2} D u\right\|_{L^{2}(|x|>1)}^{2}+2\|u\|_{L^{2}(|x|>1)}^{6}\left\|D \rho^{2}\right\|_{L^{\infty}(|x|>1)}^{2}
\end{aligned}
$$

On the other hand, we have by (3.2) and the definition of $\rho$ that

$$
\left|D \rho(x)^{2}\right| \leq \sqrt{3}|D(|x|-1)|=\sqrt{3}
$$

for $1<|x|<1+1 / \sqrt{3}$. For $|x|>1+1 / \sqrt{3}$ we also have

$$
\begin{aligned}
\left|D \rho(x)^{2}\right| & =\left|D(1-D \varphi(x))^{1 / 2}\right| \\
& =\frac{1}{2}\left|(1-D \varphi(x))^{-1 / 2} D^{2} \varphi(x)\right| \leq \frac{1}{2}\left\|D^{2} \varphi\right\|_{\infty} \leq \frac{1}{2} M .
\end{aligned}
$$

Accordingly, we have

$$
\left|D \rho(x)^{2}\right| \leq \sqrt{3}(1+M)
$$


for $|x|>1$. (3.6) and (3.7) give us

$$
\int_{|x|>1}(1-D \varphi)|u|^{6} d x \leq 8\|u\|_{L^{2}(|x|>1)}^{4}\left\|\rho^{2} D u\right\|_{L^{2}(|x|>1)}^{2}+6(1+M)^{2}\|u\|_{L^{2}(|x|>1)}^{6} .
$$

Since $\rho(x)^{4}=1-D \varphi(x),(3.5)$ and (3.8) yield

$$
\begin{aligned}
&-\operatorname{Im} \int \varphi u(t) D \bar{u}(t) d x+\int \varphi u_{0} D \bar{u}_{0} d x \\
& \leq \int_{0}^{t}\left[2 E_{0}-2\left\{1-\frac{8}{3}\|u(s)\|_{L^{2}(|x|>1)}^{4}\right\} \int_{|x|>1}(1-D \varphi)|D u(s)|^{2} d x\right. \\
&\left.+4(1+M)^{2}\|u(s)\|_{L^{2}(|x|>1)}^{6}+\frac{M}{2}\|u(s)\|_{L^{2}(|x|>1)}^{2}\right] d s .
\end{aligned}
$$

By (1.3), (3.3), and (3.9) we obtain (3.4). Q.E.D.

Now we will prove the theorem.

Proof of theorem. We assume that the solution $u(t)$ of $(1.1)-(1.2)$ exists for all $t \geq 0$ and derive a contradiction. We divide the proof into two steps.

Step 1. In this step we assume that the initial data $u_{0}$ satisfies

$$
\begin{gathered}
\eta=-2 E_{0}-4(1+M)^{2}\left\|u_{0}\right\|_{2}^{6}-\frac{M}{2}\left\|u_{0}\right\|_{2}^{2}>0, \\
\sqrt{2}\left(\int \Phi\left|u_{0}\right|^{2} d x\right)^{1 / 2}\left(\frac{2}{\eta}\left\|D u_{0}\right\|_{2}^{2}+1\right)^{1 / 2} \leq \frac{1}{2} a_{0},
\end{gathered}
$$

where $M$ and $a_{0}$ are defined as in Proposition 3.1.

We first show that if the initial data $u_{0}$ satisfies (3.10) and (3.11), then $u(t)$ satisfies (3.3) for all $t \geq 0$. We prove this by contradiction. Since $\eta>0$ and $1 \leq 2 \Phi(x)$ for $|x|>1$, we have by (3.11)

$$
\left\|u_{0}\right\|_{L^{2}(|x|>1)} \leq \frac{1}{2} a_{0} .
$$

We define $T_{0}$ as follows:

$$
T_{0}=\sup \left\{t>0 ;\|u(s)\|_{L^{2}(|x|>1)} \leq a_{0}, 0 \leq s<t\right\} .
$$

By (3.12) and the continuity in $L^{2}$ of $u(t)$, we note that $T_{0}>0$. If $T_{0}=\infty$, then the desired conclusion holds. Suppose that $T_{0}<\infty$. By the continuity in $L^{2}$ of $u(t)$, we have

$$
\left\|u\left(T_{0}\right)\right\|_{L^{2}(|x|>1)}=a_{0} .
$$

On the other hand, $u(t)$ satisfies all the assumptions in Proposition 3.1 on $\left[0, T_{0}\right)$. Therefore, by (2.5), (3.10), and Proposition 3.1 we have

$$
\int \Phi|u(t)|^{2} d x \leq \int \Phi\left|u_{0}\right|^{2} d x-2 t \operatorname{Im} \int \varphi u_{0} D \bar{u}_{0} d x-\eta t^{2}, \quad 0 \leq t<T_{0} .
$$


(3.14) and (3.10) yield

$$
\begin{aligned}
\int \Phi|u(t)|^{2} d x= & -\eta\left(t+\frac{1}{\eta} \operatorname{Im} \int \varphi u_{0} D \bar{u}_{0} d x\right)^{2} \\
& +\frac{1}{\eta}\left(\operatorname{Im} \int \varphi u_{0} D \bar{u}_{0} d x\right)^{2}+\int \Phi\left|u_{0}\right|^{2} d x \\
\leq & \frac{1}{\eta}\left\|\varphi u_{0}\right\|_{2}^{2}\left\|D u_{0}\right\|_{2}^{2}+\int \Phi\left|u_{0}\right|^{2} d x, \quad 0 \leq t<T_{0} .
\end{aligned}
$$

Noting that $\varphi^{2} \leq 2 \Phi$, we obtain by $(3.15)$

$$
\int \Phi|u(t)|^{2} d x \leq\left(\frac{2}{\eta}\left\|D u_{0}\right\|_{2}^{2}+1\right) \int \Phi\left|u_{0}\right|^{2} d x, \quad 0 \leq t<T_{0} .
$$

Since $1 \leq 2 \Phi(x)$ for $|x|>1,(3.11)$ and (3.16) show

$$
\|u(t)\|_{L^{2}(|x|>1)} \leq\left(2 \int \Phi|u(t)|^{2} d x\right)^{1 / 2} \leq \frac{1}{2} a_{0}, \quad 0 \leq t<T_{0} .
$$

This and the continuity in $L^{2}$ of $u(t)$ give us

$$
\left\|u\left(T_{0}\right)\right\|_{L^{2}(|x|>1)} \leq \frac{1}{2} a_{0},
$$

which is a contradiction of (3.13). Thus, if the initial data $u_{0}$ satisfies (3.10) and (3.11), then $u(t)$ satisfies (3.3) for all $t \geq 0$.

Therefore, since all the assumptions in Proposition 3.1 hold with $T=\infty$, $u(t)$ satisfies (3.14) with $T_{0}=\infty$; that is, for all $t \geq 0$. (3.14) implies that $\int \Phi|u(t)|^{2} d x$ becomes negative in finite time. This is a contradiction, because $\Phi(x)>0$ except when $x=0$. Hence, if the initial data $u_{0}$ satisfies (3.10) and (3.11), then $u(i)$ must blow up in finite time.

Step 2. In this step we prove the theorem for all the initial data with negative energy. For that purpose, we show that the scaling (3.1) transforms all the initial data with negative energy into the initial data satisfying (3.10) and (3.11). If we show this, then the proof of the theorem for the general case can be reduced to Step 1, since (1.1) is invariant under (3.1).

Let $u_{\varepsilon}(t, x)$ be defined as in (3.1). We put $u_{0 \varepsilon}=\varepsilon^{-1 / 2} u_{0}(x / \varepsilon)$. Then $u_{\varepsilon}(t)$ is also a solution of $(1.1)$ with $u_{\varepsilon}(0)=u_{0 \varepsilon}$ in $C\left([0, \infty) ; H^{1}\right)$. Furthermore, $u_{\varepsilon}(t)$ satisfies

$$
\begin{aligned}
& \left\|u_{\varepsilon}(t)\right\|_{2}=\left\|u_{0 \varepsilon}\right\|_{2}=\left\|u_{0}\right\|_{2}, \\
& E\left(u_{\varepsilon}(t)\right)=E\left(u_{0 \varepsilon}\right)=\varepsilon^{-2} E_{0}
\end{aligned}
$$

for $t \geq 0$.

Now we show that there exists an $\varepsilon>0$ such that

$$
\begin{aligned}
& \eta_{\varepsilon}=-2 E\left(u_{0 \varepsilon}\right)-4(1+M)^{2}\left\|u_{0 \varepsilon}\right\|_{2}^{6}-\frac{M}{2}\left\|u_{0 \varepsilon}\right\|_{2}^{2}>0, \\
& \sqrt{2}\left(\int \Phi\left|u_{0 \varepsilon}\right|^{2} d x\right)^{1 / 2}\left(\frac{2}{\eta_{\varepsilon}}\left\|D u_{0 \varepsilon}\right\|_{2}^{2}+1\right)^{1 / 2} \leq \frac{1}{2} a_{0} .
\end{aligned}
$$


If we choose $\varepsilon>0$ so that

$$
\varepsilon^{2}<-2 E_{0}\left[4(1+M)^{2}\left\|u_{0}\right\|_{2}^{6}+\frac{M}{2}\left\|u_{0}\right\|_{2}^{2}\right]^{-1},
$$

then we have (3.19), by (3.17) and (3.18). Since $\left\|D u_{0 \varepsilon}\right\|_{2}^{2}=\varepsilon^{-2}\left\|D u_{0}\right\|_{2}^{2}$, we have by (3.17) and (3.18) that

$$
\frac{2}{\eta_{\varepsilon}}\left\|D u_{0 \varepsilon}\right\|_{2}^{2} \leq C_{0}, \quad 0<\varepsilon<\varepsilon_{1},
$$

for some $\varepsilon_{1}>0$, where $C_{0}$ depends on $\varepsilon_{1}$ but does not depend on $\varepsilon$. On the other hand, Lemma 2.3 shows that there exists an $\varepsilon_{2}>0$ such that $\varepsilon_{2}<\varepsilon_{1}$ and

$$
\int \Phi\left|u_{o \varepsilon}\right|^{2} d x \leq 2\left\|R u_{0 \varepsilon}\right\|_{2}^{2} \leq \frac{1}{8}\left(C_{0}+1\right)^{-1} a_{0}^{2}
$$

for $0<\varepsilon<\varepsilon_{2}$, where $R$ is the function defined in Lemma 2.3. (3.22) assures that (3.20) holds for $0<\varepsilon<\varepsilon_{2}$. Therefore, if we choose $\varepsilon>0$ satisfying (3.21) and $\varepsilon<\varepsilon_{2}, u_{0 \varepsilon}$ satisfies (3.19) and (3.20).

Thus, we can reduce the proof of the theorem for the general case to Step 1, when we consider $u_{\varepsilon}(t, x)$ instead of $u(t, x)$. This completes the proof of the theorem. Q.E.D.

\section{REFERENCES}

1. T. Cazenave and F. B. Weissler, The Cauchy problem for the nonlinear Schrödinger equation in $H^{1}$, Manuscripta Math. 61 (1988), 477-498.

2. _ The structure of solutions to the pseudoconformally invariant nonlinear Schrödinger equation, Proc. Roy. Sci. Edinburgh (to appear).

3. L. M. Degtyarev, V. E. Zakharov, and L. I. Rudakov, Two examples of Langmuir wave collapse, Soviet Phys. JETP 41 (1975), 57-61.

4. J. Ginibre and G. Velo, On a class of nonlinear Schrödinger equations I: The Cauchy problem, II: Scattering theory, general case, J. Funct. Anal. 32 (1979), 1-71.

5. R. T. Glassey, On the blowing-up of solutions to the Cauchy problem for the nonlinear Schrödinger equation, J. Math. Phys. 18 (1977), 1794-1797.

6. T. Kato, On nonlinear Schrödinger equations, Ann. Inst. H. Poincaré Phys. Théor. 46 (1987), 113-129.

7. __ Nonlinear Schrödinger equations, preprint.

8. O. Kavian, A remark on the blow-up of solutions to the Cauchy problem for nonlinear Schrödinger equations, Trans. Amer. Math. Soc. 299 (1987), 193-203.

9. B. LeMesurier, G. Papanicolaou, C. Sulem, and P. L. Sulem, The focusing singularity of the nonlinear Schrödinger equation, in Direction in partial differential equations (M. G. Crandall, P. H. Rabinowitz, and R. E. Turner, eds.), Academic Press, New York, 1987, pp. 159-201.

10. J. E. Lin and W. A. Strauss, Decay and scattering of solutions of a nonlinear Schrödinger equation, J. Funct. Anal. 30 (1978), 245-263.

11. F. Merle, Limit of the solution of the nonlinear Schrödinger equation at the blow-up time, J. Funct. Anal. 84 (1989), 201-214.

12. _ Construction of solutions with exactly $k$ blow-up points for the Schrödinger equation with critical nonlinearity, Comment. Math Phys. 129 (1990), 223-240. 
13. F. Merle and Y. Tsutsumi, $L^{2}$ concentration of blow-up solutions for the nonlinear Schrödinger equation with critical power nonlinearity, J. Differential Equations 84 (1990), 205214.

14. H. Nawa, "Mass" concentration phenomenon for the nonlinear Schrödinger equation with the critical power nonlinearity I, II, preprint.

15. H. Nawa and M. Tsutsumi, On blow-up for the pseudo-conformally invariant nonlinear Schrödinger equation, Funkcialaj Ekvacioj 32 (1989), 417-428.

16. T. Ogawa and Y. Tsutsumi, Blow-up of $H^{1}$ solution for the nonlinear Schrödinger equation, J. Differential Equations (to appear).

17. W. A. Strauss, Existence of solitary waves in higher dimensions, Comm. Math. Phys. 55 (1977), 149-162.

18. __ Nonlinear wave equations, CBMS Regional Conference Series in Mathematics, no. 73, Amer. Math. Soc., Providence, RI, 1989.

19. M. Tsutsumi, Nonexistence of global solutions to the Cauchy problem for the damped nonlinear Schrödinger equations, SIAM J. Math. Anal. 15 (1984), 357-366.

20. Y. Tsutsumi, Rate of $L^{2}$ concentration of blow-up solutions for the nonlinear Schrödinger equation with critical power nonlinearity, Nonlinear Anal. (to appear).

21. M. I. Weinstein, Nonlinear Schrödinger equations and sharp interpolation estimates, Comm. Math. Phys. 87 (1983), 567-576.

22. __ On the structure and formation of singularities in solutions to nonlinear dispersive evolution equations, Comm. Partial Differential Equations 11 (1986), 545-565.

23. - The nonlinear Schrödinger equation-Singularity formation, stability and dispersion, The Connection between Infinite and Finite Dimensional Dynamical Systems, Contemp. Math., vol. 99, Amer. Math. Soc., Providence, RI, 1989, pp. 213-232.

Department of Pure and Applied Sciences, University of ToKyo Komaba 3-8-1, MeguroKU, TOKYO 153, JAPAN

Current address: Department of Mathematics, School of Science, Nagoya University, Chikusa$\mathrm{Ku}$, Nagoya 464-01, Japan

Department of Mathematics, School of Science, Nagoya University, Chikusa-ku, NAGOYA 464-01, JAPAN 